\title{
Diseño y Construcción de un Elevador Hidráulico con Volteo para la Cosecha de Palma Africana con una Capacidad de 5 Toneladas
}

\author{
Javier Enrique Orna Chávez, MsC \\ Santiago Alejandro Lopez Ortiz, MsC \\ Edwin Fernando Viteri Nuñez, MsC \\ Diego Fernando Mayorga Perez, MsC \\ Docentes, Facultad de Mecánica \\ Escuela Superior Politécnica de Chimborazo, Ecuador \\ Diego Armando Analuisa Topa, Eng \\ Julio César Jácome Iza, Eng \\ Egresados, Facultad de Mecánica \\ Escuela Superior Politécnica de Chimborazo, Ecuador
}

Doi: 10.19044/esj.2019.v15n9p536 URL:http://dx.doi.org/10.19044/esj.2019.v15n9p536

\section{Resumen}

Tomando en cuenta la información recopilada mediante un estudio bibliográfico acerca de la palma africana, sus características, propiedades, metodologías de cosecha y trasporte, es posible determinar que el problema a tratar son los tiempos de cosechado del fruto de dicha palma. Por consiguiente, el presente trabajo tiene como alcance diseñar y construir un elevador hidráulico con volteo para la cosecha de palma africana con una capacidad de 5 toneladas para de esta manera aumentar la eficiencia y la producción de las empresas palmicultoras. Esto implicó la recopilación de información necesaria para realizar la metodología de diseño, realizando un estudio de alternativas existentes en el mercado para luego seleccionar aquellq que sea más viable y que cumpla con nuestros requerimientos y parámetros de calidad. Posteriormente se realizó el diseño mecánico e hidráulico que conforma el elevador hidráulico, realizando cálculos mediante elemento finito analíticamente y se comprobó el diseño en el software ANSYS. En el análisis estático de la estructura en posición baja, media y alta se determina que la parte más crítica se encuentra en la posición alta. Para completar el diseño mecánico se realizó el análisis dinámico de prueba de vuelco en el software ADAMS. Finalmente se seleccionaron las partes hidráulicas de acuerdo a la presión que tiene la bomba hidráulica del tractor New Holland. En conclusión, el elevador hidráulico con volteo soporta una 
capacidad de carga de 6,5 toneladas y tiene un sistema de transporte adecuado lo cual garantiza una producción eficiente en la cosecha de palma africana.

Palabras claves: Tecnología, capacidad de carga, sistema de dirección, sistema hidráulico, ángulo de volteo

\title{
Design and Construction of a Hydraulic Elevator with Turning for the Harvest of the African Palm with a Capacity of 5 Tons
}

Javier Enrique Orna Chávez, MsC

Santiago Alejandro Lopez Ortiz, MsC

Edwin Fernando Viteri Nuñez, MsC

Diego Fernando Mayorga Perez, MsC

Docentes, Facultad de Mecánica

Escuela Superior Politécnica de Chimborazo, Ecuador

Diego Armando Analuisa Topa, Eng

Julio César Jácome Iza, Eng

Egresados, Facultad de Mecánica

Escuela Superior Politécnica de Chimborazo, Ecuador

\begin{abstract}
Taking into account the information collected through a bibliographic study about African palm, its characteristics, properties, harvesting and transport methodologies, it is possible to determine that the problem to be treated are the harvested times of the fruit of the said palm. Therefore, this paper focuses on designing and building a hydraulic lift with overturning for the harvest of African palm with a capacity of 5 tons in order to increase the efficiency and production of palm oil companies. This involved the collection of information necessary to carry out the design methodology, carrying out a study of existing alternatives in the market, and then selecting which one is more viable and which meets our requirements and quality parameters. Subsequently, the mechanical and hydraulic design that forms the hydraulic lift was carried out. It performs finite element calculations analytically and the design was checked using the ANSYS software. In the static analysis of the structure in low, medium and high position, it is determined that the most critical part is in the high position. To complete the mechanical design, the
\end{abstract}


dynamic rollover test analysis was performed using the ADAMS software. Finally the hydraulic parts were selected according to the pressure of the hydraulic pump of the New Holland tractor. In conclusion, the hydraulic lift with tilting supports a load capacity of 6.5 tons and have an adequate transportation system which guarantees efficient production in the African palm harvest.

Keywords: Technology, load capacity, steering system, hydraulic system, tipping angle

\section{Introduction}

En la actualidad, el cultivo de palma africana es uno de los principales cultivos en el país debido a los múltiples usos de esta planta así como también a su uso como biocombustible. La Elaeis guineensises un fruto rico en aceite que se cultiva principalmente en las provincias de Esmeraldas, Los Ríos, Santo Domingo, Sucumbíos y Orellana (Fats, 2015). La palma africana (Elaeisguineensis) es originaria del Golfo de Guinea (África occidental) y se extiende hasta $15^{\circ}$ de latitud norte y sur. Es una planta perenne, alcanzando más de 100 años, pero bajo cultivo sólo se le permite llegar hasta los 25 años, que es cuando alcanza los $12 \mathrm{~m}$ de altura. En su estado natural llega a superar los 40 metros (Genty, 2011).

El aceite representa el $35 \%$ del total de grasas vegetales y animales producidas en el mundo, porcentaje dentro del cual se encuentra el aceite de palma. La mayoría del aceite de palma se exporta por el Puerto de Esmeraldas, lugar donde se han montado bodegas para el almacenamiento del producto.

Tabla 1. Principales Países productores y provincias de Palma de Aceite en el Ecuador

\begin{tabular}{|l|c|c|}
\hline \multicolumn{1}{|c|}{ PAIS } & SUPERFICIE SEMBRADA & PRODUCCIÓN \\
\hline Indonesia & $5^{\prime} 500,000$ Has. & 20,9 millones TM de Aceite \\
Malasia & $4^{\prime} 600,00$ Has. & 17,6 millones TM de Aceite \\
Tailandia & 420,000 Has. & 1,3 millones TM de Aceite \\
Nigeria & 380,000 Has. & 794 mil TM de Aceite \\
Papúa N.G. & 134,000 Has. & 470 mil TM de Aceite \\
Colombia & 450,000 Has. & 900 mil TM de Aceite \\
Ecuador & 250,000 Has. & 436 mil TM de Aceite \\
Resto del Mundo & $1^{\prime} 200,000$ Has. & 4,0 millones TM de Aceite $(6,15 \%)$ \\
\hline
\end{tabular}




\begin{tabular}{|c|c|c|c|c|c|}
\hline Año & Provincia & $\begin{array}{l}\text { Superficie } \\
\text { Cosechada } \\
\text { (ha) }\end{array}$ & $\begin{array}{l}\text { Producción } \\
\text { (TM.) }\end{array}$ & $\begin{array}{l}\text { Rendimiento } \\
\text { (TM/ha) }\end{array}$ & $\begin{array}{l}\text { Porcentaje } \\
\text { Nacional }\end{array}$ \\
\hline \multirow{5}{*}{2016} & ESMERALDAS & 138,566 & $1,584,758$ & 11.44 & $50.73 \%$ \\
\hline & LOS RÍOS & 27,256 & 412,024 & 15.12 & $13.19 \%$ \\
\hline & $\begin{array}{l}\text { SANTO } \\
\text { DOMINGO }\end{array}$ & 21,399 & 272,519 & 12.74 & $8.72 \%$ \\
\hline & $\begin{array}{l}\text { RESTO DE } \\
\text { PROVINCIAS }\end{array}$ & 76,299 & 854,768 & 11.20 & $27.36 \%$ \\
\hline & Total general & $263,520.00$ & $3,124,069.00$ & 11.86 & $100 \%$ \\
\hline
\end{tabular}

Fuente: Investigación bibliográfica, 2018

Realizado por: Los autores.

Hace cinco años, la palma aceitera comenzó a ganar terreno. Por su composición física, el aceite de palma puede usarse en diversas preparaciones sin necesidad de hidrogenarse, proceso mediante el cual se forman los trans, indeseables ácidos grasos precursores de enfermedades como la diabetes y los cardiovasculares, entre otras. Actualmente, es el segundo aceite más consumido en el mundo y se emplea como aceite de cocina, para elaborar productos de panadería, pastelería, confitería, heladería, sopas instantáneas, salsas, diversos platos congelados y deshidratados, cremas no lácteas para mezclar con el café (Chinchilla, 2016). En pocas palabras, el panorama está bastante claro: la palma "no es un negocio para campesinos sino para grandes empresas". Sin embargo, en la actualidad la rentabilidad del producto se ve reflejada en los sembríos de los pequeños productores.

La palma es uno de los cultivos de más rápida expansión a nivel mundial. En los últimos 50 años se ha cultivado en 43 países, siendo los más grandes exportadores de aceite de palma Malasia e Indonesia, seguido por países latinoamericanos como Ecuador y Colombia (Mexrón, 1997). Ecuador se encuentra entre los 10 mejores en la producción de aceite de palma en el mundo. El cultivo y procesamiento de palma genera en el país más de $150 \mathrm{mil}$ empleos (PWC, 2011). Como se puede notar, el cosechado de la palma africana es un proceso de gran importancia, por lo cual son necesarios sistemas de cosechado inmediatos para obtener un aceite de calidad (Urueta, 2018).

\section{Procesos y tecnologías de cosechado}

Existen diferentes tipos de vehículos que son utilizados para trasportar los racimos de fruta fresca desde el campo hasta la planta de beneficio, entre ellos: tractomulas, camiones de carrocería, camiones de autocarga y tractores con góndolas. La elección del vehículo a utilizar depende de factores como el estado de la infraestructura vial, capacidad de trasporte y de la distancia a recorrer (Fontanilla, 2015). 
Ante la oportunidad de maximizar el volumen de racimos de frutas frescas transportados por vehículo al día y disminuir el número de golpes que recibe se opta por llevarlos en vehículos de carga y descarga rápida. Los vehículos que no poseen sistemas de descargue rápido presentan tiempos muertos para su llenado (que generalmente se hace manual) y deben esperar hasta que la planta les asigne el turno de descargue; cabe aclarar que este tiempo se incrementa si en los centros de acopio no existen tolvas de cargue y si en las plantas de beneficio los patios de recibo de frutos son pequeños o carecen de plataformas hidráulicas que inclinen los vehículos para que los racimos de fruto fresco se descarguen por gravedad (Fontanilla, 2015).

\section{Cosechado de la palma africana comúnmente}

Los tipos de cosechado que se dan en las haciendas son: carretas por tracción animal y con pequeñas estructuras sobre animales. El proceso de cosecha de palma africana se realiza dos veces al mes por cada lote. Este proceso es realizado por 3 trabajadores: el cortador de fruta, el recogedor de fruta y el transportador de fruta (Bula, 2008). Su importancia radica en construir un cultivo agroindustrial que ocupa una significativa cantidad de mano de obra, tanto en la fase de campo, como en su fase productiva y de industrialización. Actualmente, en Ecuador las investigaciones han avanzado hasta el punto de encontrar nuevas variedades híbridas, gracias alas cuales se puede tener una buena producción en tamaño, en número de racimos, formas de cosechado al igual que una importante cantidad de porcentaje de extracción de aceite (Sanabria, 2018).

Carretas por tracción animal. Este tipo de cosechado se realiza por medio de tracción animal, en donde estos son forzados al trabajo duro todo el día con un tiempo de cosecha muy alto por lo que se ve afectada la producción de palma africana.

Transporte con pequeñas estructuras en animales. Para la aplicación de este tipo de trasporte los animales tienen una pequeña estructura que llevan según se va cosechando. La cosecha es demorosa, y se ve una notable fatiga de los animales, puesto que son expuestos a trabajos forzados para la cosecha del grano de fruto fresco de palma africana. 
Figura 1. Forma de cosechado del fruto por tracción animal.
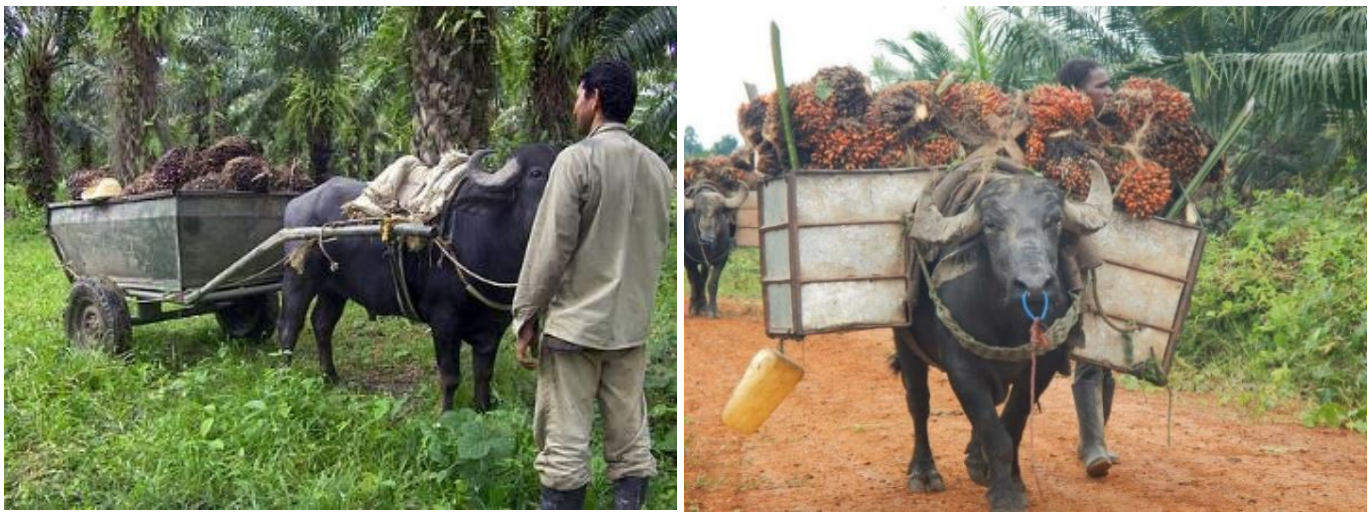

Fuente: Investigación bibliográfica, 2018

Realizado por: Los autores.

\section{Tipos de transporte de la palma Africana}

Esta etapa del proceso está compuesta por la maquinaria y equipo de operarios constituidos de tres funciones: uno de ellos es el operador y los otros dos son ayudantes que serán responsables de recoger los frutos. Como asistencia en esta recolección se utiliza una herramienta de soporte, la cual sirve para apalancar el racimo dentro del remolque hidráulico. Una vez recolectado los frutos, se acarrean hasta los contenedores de forma estratégica a lo largo de las vías de la plantación (Ocampo, 2018).

Figura 2. Sistema de recolección de frutos del interior de las parcelas.(A) Equipo entrando

(B) Recolección de racimos (C) Salida de la parcela al camino del contenedor.
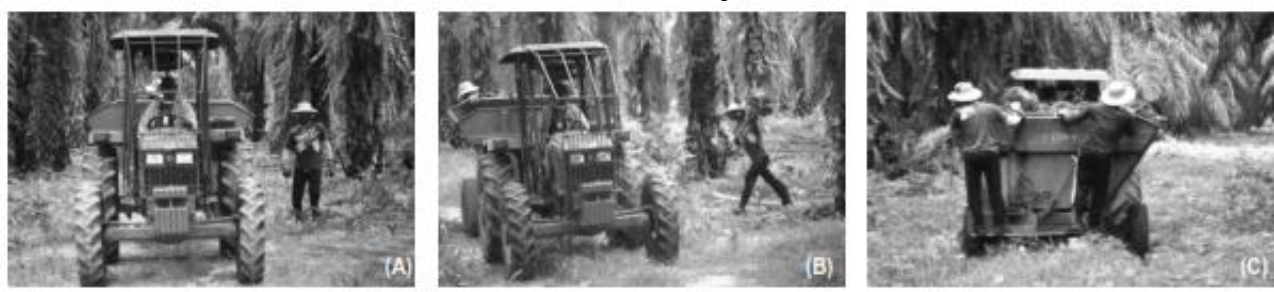

Fuente: Investigación bibliográfica, 2018

Realizado por: Los autores.

\section{Requerimientos del elevador hidráulico}

Voz del usuario. La voz del usuario representa los requerimientos básicos y las características con las que debe contar el elevador hidráulico con volteo para la cosecha de palma africana. Estas características son las indicadas a continuación:

- Dimensiones adecuadas,

- Capacidad de 5 ton,

- Fácil mantenimiento,

- Llantas de trasporte con dirección, 
- Altura mínima de $3 \mathrm{~m}$,

- Costo de la máquina,

- Máquina ligera,

- Descargue total de la fruta,

- Fácil operación,

- Sistema de elevación hidráulico eficiente.

\section{Metodología}

En el presente artículo se utilizaron técnicas de investigación bibliográfica y de campo, para llevar a cabo el diseño del elevador hidráulico. La máquina debe contar con las especificaciones y requerimientos, satisfaciendo las necesidades del usuario cuya actividad se ve reflejada elevando la cantidad requerida sin ningún inconveniente.

El diseño y construcción del elevador hidráulico se basó en fases e interacciones del proceso de diseño. Las alternativas son las siguientes:

Alternativa 1. Se observa que la tolva tiene unas dimensiones grandes para depositar en camiones con baldes de la misma dimensión. Para camiones con baldes pequeños no será posible la descarga total del fruto de palma africana pues puede caer al suelo por las dimensiones de dicha tolva del elevador.

Ventajas: Rápida descarga del fruto de palma africana.

Desventajas: Sistema de volteo inestable.

Aplicar grasa en las carrileras cada vez que se utiliza la máquina.

Alternativa 2. Se observa que es un sistema hidráulico de elevación tipo tijera con araña y no tiene posibilidades de giro en caso de ser necesario. Razón por la cual no cumple con nuestros requerimientos.

Ventajas: $\quad$ El sistema de volteo es estable.

Desventajas: No tiene sistema de dirección para el giro de las llantas de la máquina. Se recalienta la barra donde está el mecanismo para elevación de la máquina. Aplicar grasa en las carrileras cada vez que se utiliza la máquina.

Alternativa 3. Se trata de un sistema hidráulico de elevación tipo tijera con dos cilindros y un sistema de dirección que puede girar sin ningún problema lo cual nos garantiza un funcionamiento óptimo.

Ventajas: Rápida descarga del fruto de palma africana.

El sistema de volteo es estable. Tiene sistema de dirección para el giro de las llantas de la máquina.

Desventajas: Aplicar grasa en las carrileras para utilizar la máquina. 
Figura 3. Alternativa 1, Alternativa 2 y Alternativa 3 respectivamente
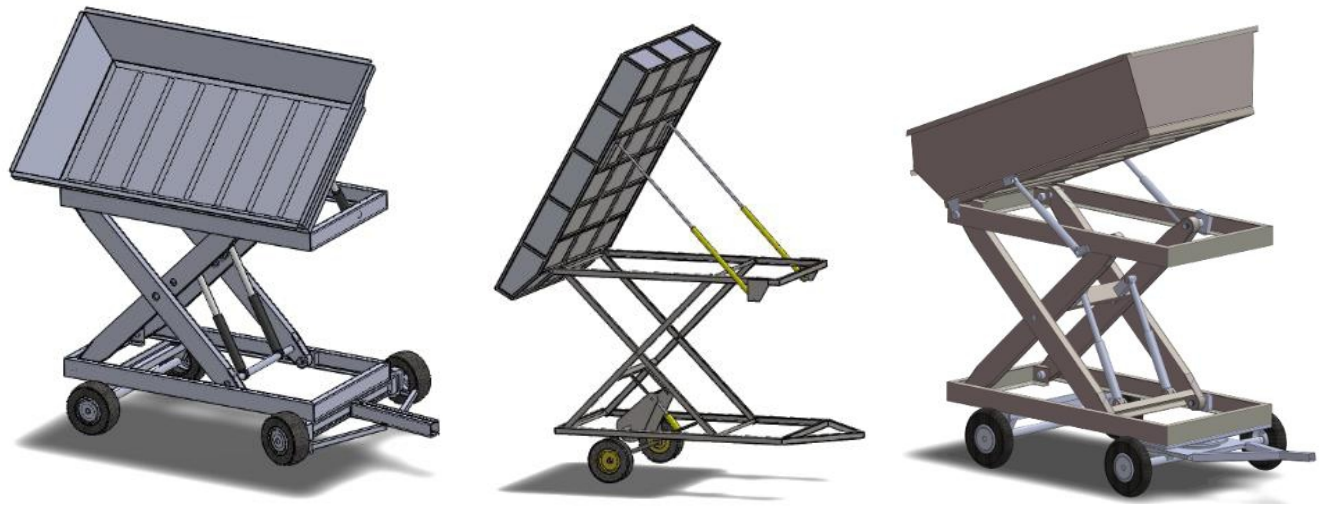

Fuente: Solidworks, 2018

Realizado por: Los autores.

\section{Materiales y Métodos}

Las características generales del elevador hidráulico se estiman en la tabla correspondiente y se utilizan para el desarrollo y aplicación adecuada de una correcta metodología de diseño.

Tabla 2. Características del elevador hidráulico

\begin{tabular}{|l|l|}
\hline \multicolumn{2}{|c|}{ Elevador hidráulico con volteo } \\
\hline \multirow{5}{*}{ Características } & $\begin{array}{l}\text { Dimensiones generales: } \\
\text { Alto }=1,50 \mathrm{~m} \\
\text { Ancho }=1.60 \mathrm{~m} \\
\text { Largo }=4 \mathrm{~m}\end{array}$ \\
\cline { 2 - 3 } & Capacidad de carga $=$ hasta \\
& 6500 Kg \\
\cline { 2 - 3 } & Material de construcción \\
& Acero estructural A-36 \\
& Acero laminado en frio A-36 \\
\cline { 2 - 3 } & Remolcado por tractor \\
& agrícola. \\
& NEW HOLLAND \\
& AGRICULTURE \\
\cline { 2 - 3 } & Peso total del elevador $=3221$ \\
& Kg \\
\cline { 2 - 3 } & Frontal con dirección: tipo \\
& camión con barras macizas \\
& para direccionar. \\
\hline
\end{tabular}

Fuente: Investigación bibliográfica, 2018

Realizado por: Los autores.

\section{Modelado geométrico}

Con la ayuda de herramientas CAD se realizó el modelado geométrico de las piezas que conforman el elevador como se observa en la siguiente figura. 
Figura 4. Modelado del elevador hidráulico con volteo

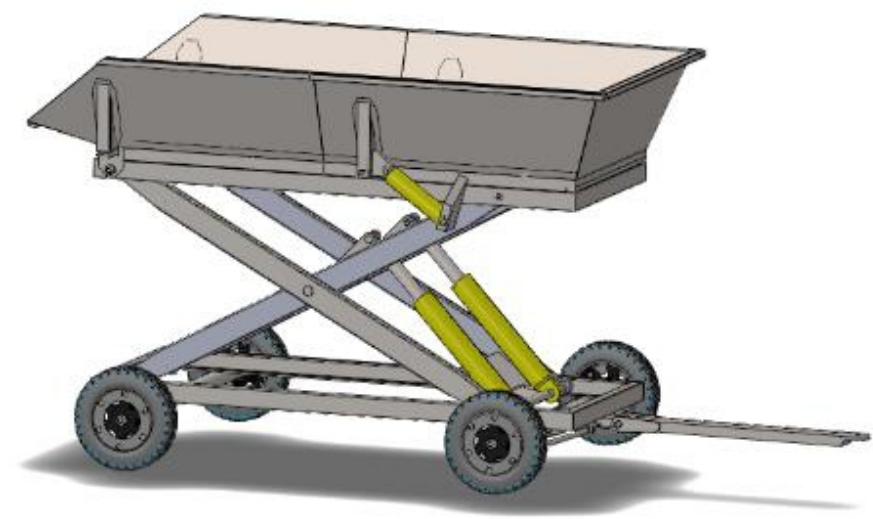

Fuente: Solidworks, 2018

Realizado por: Los autores.

\section{Diseño mecánico de la tolva}

El dimensionamiento de los elementos del elevador va acorde con el peso del fruto y las dimensiones. Para el presente análisis se determinaron como elementos críticos la tolva y el sistema de elevación tipo tijera, el cual se acopla a las carrileras conjuntamente con el sistema de transporte. En estos elemento es donde se aplica la carga durante el proceso de elevación y vaciado hacia los camiones que transportan el fruto a las plantas procesadoras.

La presión aplicada en la tolva es de es de 7,65625 KPa en el eje negativo de " $y$ ", el peso de carga es de 5 toneladas por el área critica que se encuentra en la parte inferior de la misma.

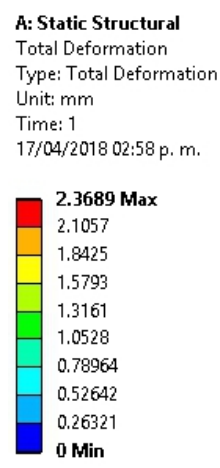

Figura 5. Carga aplicada en la tolva

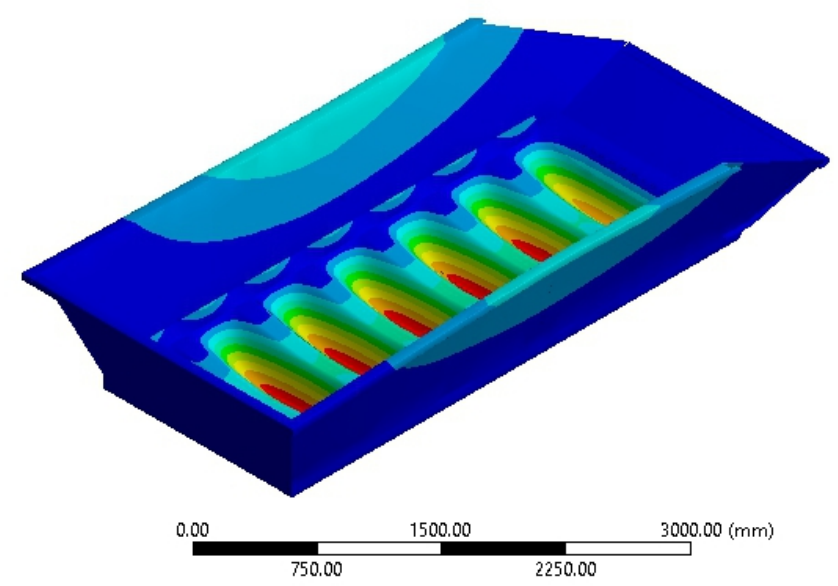

Fuente: Ansys, 2018

Realizado por: Los autores. 
Deformación total. Con los resultados alcanzados por el software es posible observar que la deformación total de la tolva es $2.39 \mathrm{~mm}$, lo cual nos indica una solución aceptable para nuestro diseño. En la siguiente tabla se indican los valores de refinamiento de la malla en el software ANSYS para obtener valores estables.

Tabla 3. Refinamiento de la malla respecto a la deformación de la tolva

\begin{tabular}{|c|c|c|c|}
\hline $\begin{array}{c}\text { Tamaño de } \\
\text { malla (mm) }\end{array}$ & Deformación(mm) & $\begin{array}{c}\text { Número de } \\
\text { Elementos }\end{array}$ & $\begin{array}{c}\text { Número } \\
\text { de Nodos }\end{array}$ \\
\hline 10 & 2.3815 & 77115 & 481281 \\
\hline 9 & 2.3821 & 91809 & 584619 \\
\hline 8 & 2.3727 & 113127 & 734475 \\
\hline 7 & 2.3799 & 143265 & 946221 \\
\hline 6 & 2.3939 & 189933 & 1273947 \\
\hline 5 & 2.3949 & 220857 & 1491030 \\
\hline
\end{tabular}

Fuente: Ansys, 2018

Realizado por: Los autores.

Una vez adquiridos los resultados procedemos a realizar la curva de convergencia para observar dónde nuestra deformación es estable, indicándonos así una solución correcta.

Figura 6. Deformación vs Número de elementos de la tolva

Deformación vs Número de Elementos

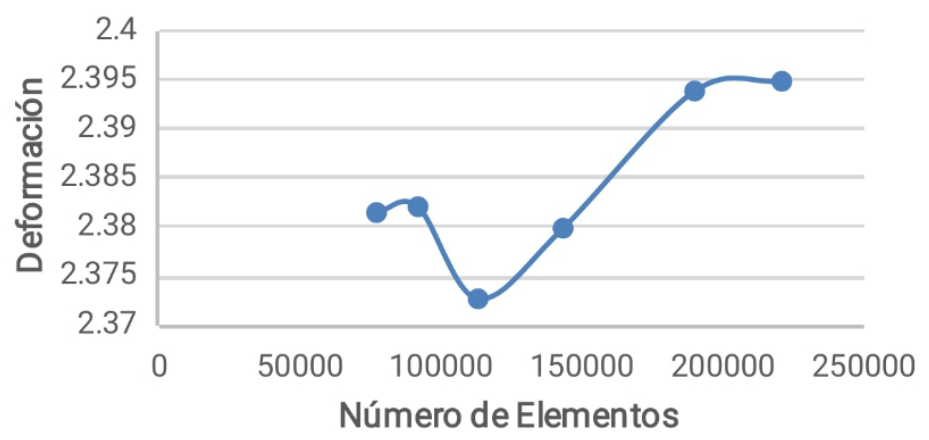

Fuente: Ansys, 2018

Realizado por: Los autores.

Factor de seguridad. Cumpliendo con los parámetros anteriores obtenemos un factor de seguridad de 1.33, lo cual nos garantiza la confiabilidad de nuestro diseño.

En la siguiente tabla se indican los valores de refinamiento de la malla para obtener valores estables. 
Tabla 4. Refinamiento de la malla respecto al factor de seguridad de la tolva.

\begin{tabular}{|c|c|c|c|}
\hline $\begin{array}{c}\text { Tamaño de } \\
\text { malla }(\mathrm{mm})\end{array}$ & $\begin{array}{c}\text { Factor de } \\
\text { seguridad }\end{array}$ & $\begin{array}{c}\text { Número de } \\
\text { Elementos }\end{array}$ & $\begin{array}{c}\text { Número de } \\
\text { Nodos }\end{array}$ \\
\hline 10 & 1.3398 & 77115 & 481281 \\
\hline 9 & 1.3377 & 91809 & 584619 \\
\hline 8 & 1.3517 & 113127 & 734475 \\
\hline 7 & 1.3399 & 143265 & 946221 \\
\hline 6 & 1.3375 & 189933 & 1273947 \\
\hline 5 & 1.3376 & 220857 & 1491030 \\
\hline
\end{tabular}

Fuente: Ansys, 2018

Realizado por: Los autores.

Una vez adquiridos los resultados procedemos a realizar la curva de convergencia para observar donde nuestro factor de seguridad es estable indicándonos una solución correcta.

Figura 7. Factor de seguridad vs Número de elementos de la tolva

\section{Factor de seguridad vs Número de Elementos}

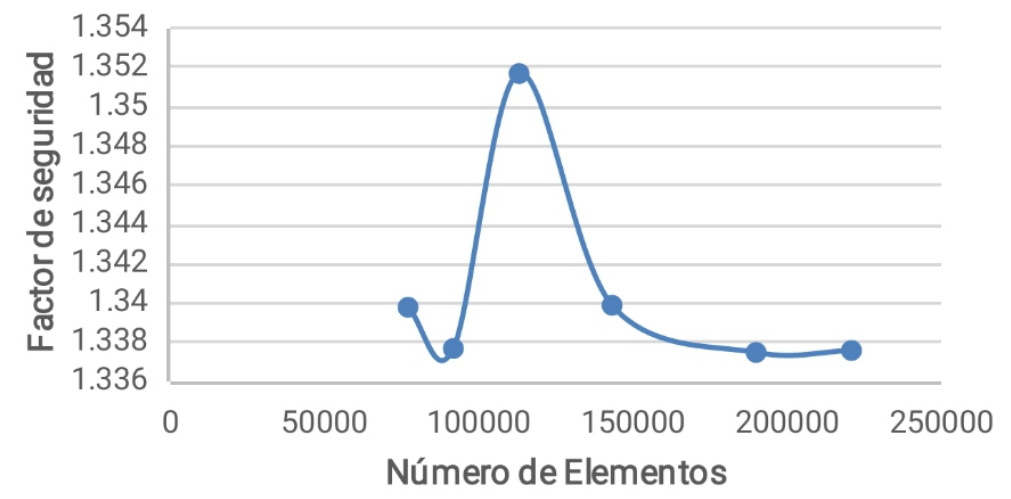

Fuente: Ansys, 2018

Realizado por: Los autores.

\section{Cálculo y análisis estructural del sistema de elevación tipo tijera}

Para realizar un análisis de la estructura del elevador consideramos el peso de la tolva y peso de carga de $5000 \mathrm{~kg}$, la cual se distribuye uniformemente en el elevador. Realizamos un análisis estático en posición máxima, media y baja para encontrar el punto crítico de la estructura del elevador, y por medio del software SolidWorks realizamos el dimensionamiento de la estructura para realizar el respectivo cálculo estático y dinámico. 
Figura 8. Esquemas de la estructura en posición alta, media y baja respectivamente
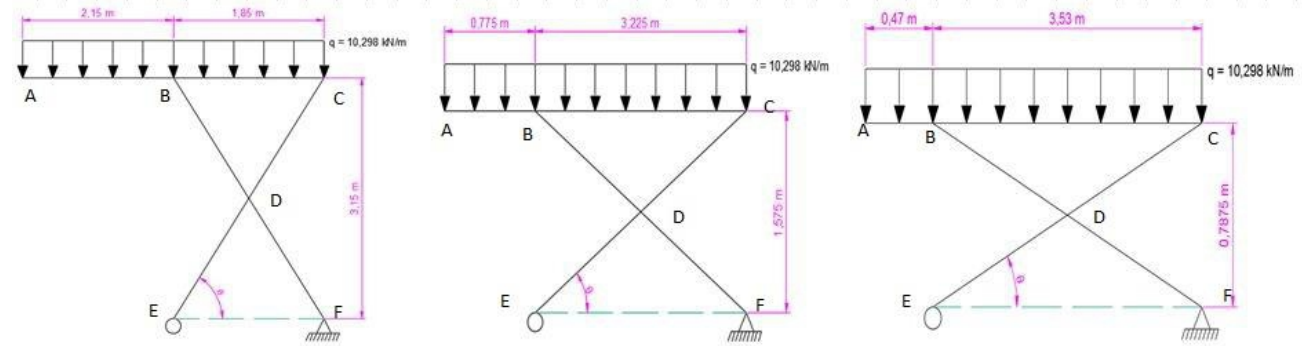

Fuente: Autocad, 2018

Realizado por: Los autores.

A partir de los diagramas de la estructura y el análisis estático en diferentes posiciones y por medio de ecuaciones como la sumatoria de fuerzas se determinan las fuerzas para cada punto.

Tabla 5. Fuerzas para cada posición de la estructura del elevador

\begin{tabular}{|l|l|l|}
\hline \multicolumn{1}{|c|}{ Posición alta } & Posición media & \multicolumn{1}{c|}{ Posición baja } \\
\hline $\mathrm{F}_{\mathrm{DE}}=51,54 \mathrm{kN}$ & $\mathrm{F}_{\mathrm{DE}}=58,20 \mathrm{kN}$ & $\mathrm{F}_{\mathrm{ED}}=107,25 \mathrm{kN}$ \\
$\mathrm{F}_{\mathrm{FD}}=3,83 \mathrm{kN}$ & $\mathrm{F}_{\mathrm{FD}}=35,66 \mathrm{kN}$ & $\mathrm{F}_{\mathrm{FD}}=82,02 \mathrm{kN}$ \\
$\mathrm{F}_{\mathrm{DC}}=51,54 \mathrm{kN}$ & $\mathrm{F}_{\mathrm{DC}}=61,2 \mathrm{kN}$ & $\mathrm{F}_{\mathrm{DC}}=107,25 \mathrm{kN}$ \\
$\mathrm{F}_{\mathrm{DB}}=3,83 \mathrm{kN}$ & $\mathrm{F}_{\mathrm{DB}}=38,66 \mathrm{kN}$ & $\mathrm{F}_{\mathrm{DB}}=82,02 \mathrm{kN}$ \\
\hline
\end{tabular}

Realizado por: Los autores.

\section{Análisis en el software ANSYS de la estructura}

Una vez realizado el análisis estático del elevador en posición alta, media y baja notamos que necesitamos una fuerza superior a $107,25 \mathrm{kN}$ para subir el elevador hidráulico. La parte más crítica se encuentra en la posición alta, punto donde realizaremos un análisis estructural en el software ANSYS para comprobar la resistencia de la estructura del levador.

Figura 9. Carga aplicada en la estructura del elevador hidráulico

A: Static Structur

Remote Force

Time: 1.s $17 / 04 / 201802: 36$ p. m.

A Fixed Support

A Fixed Support

C Remote Force: $41192 \mathrm{~N}$

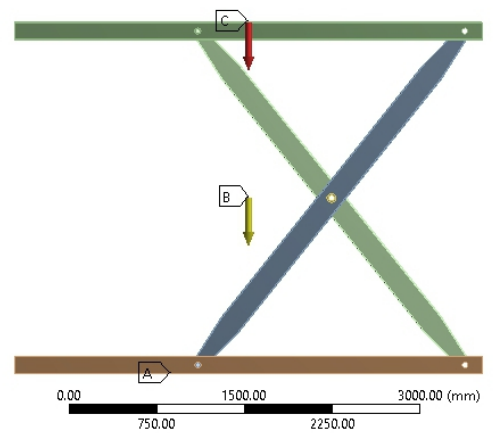

Fuente: Ansys, 2018

Realizado por: Los autores. 
Una vez alcanzados los resultados procedemos a realizar la curva de convergencia donde se observa una deformación de $6 \mathrm{~mm}$ aproximadamente de toda la estructura en dicha posición.

Figura 10. Convergencia de la deformación vs número de elementos de la estructura.

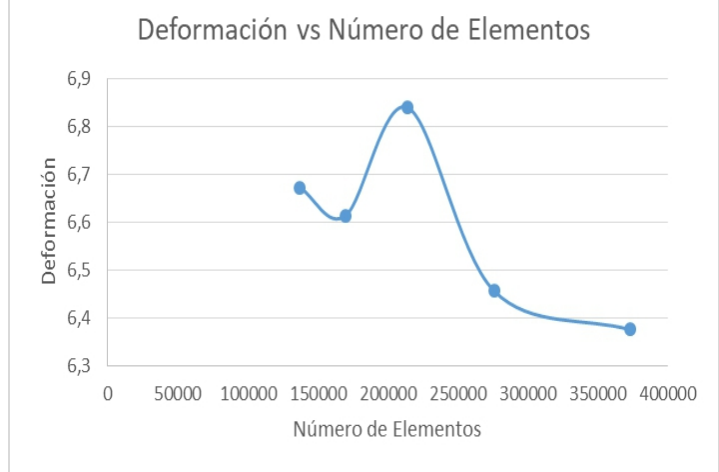

Fuente: Ansys, 2018

Realizado por: Los autores.

Factor de seguridad. Cumpliendo con los parámetros anteriores obtenemos un factor de seguridad de $\eta=1.6151$, lo cual nos garantiza la confiabilidad de nuestro diseño.

\section{Análisis dinámico de la estructura del elevador hidráulico}

Una vez realizado el análisis estático de la estructura del elevador hidráulico procedemos a verificar el movimiento de la máquina. Acto seguido procedemos a colocar el peso de la tolva y la carga total que soporta la estructura para verificar el movimiento, siendo la carga total $80378 \mathrm{~N}$.

Figura 10. Factor de seguridad y sistemas de elevación dinámico del sistema de elevación en el software ANSYS
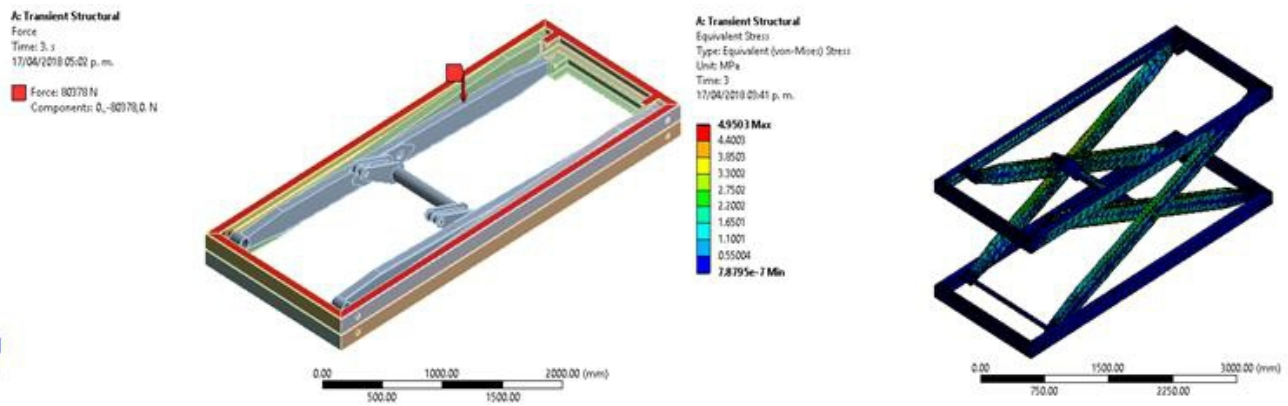

Fuente: Ansys, 2018

Realizado por: Los autores.

Validación de la estructura mediante el método analítico

Datos:

$$
F_{D C}=107,25 \mathrm{kN}
$$


Donde:

$$
\mathrm{d}=3 \text { in }=76,2 \mathrm{~mm}
$$

$\mathrm{F}_{\mathrm{DC}}=$ Fuerza máxima en el nodo " $\mathrm{D}$ " de la parte inferior de la estructura del elevador hidráulico.

d = Diámetro del bocín en las articulaciones

$$
\begin{aligned}
& \sigma=\frac{F_{D C}}{A}=\frac{F_{D C}}{\frac{\pi}{4} * d^{2}} \\
& \sigma=23,51 \mathrm{MPa}
\end{aligned}
$$

Analíticamente

El esfuerzo máximo en la parte inferior del elevador de la estructura analíticamente es $23,51 \mathrm{MPa}$ y el esfuerzo máximo obtenido mediante Transient Structural (software ANSYS) es 19,116 MPa, lo cual valida la estructura indicando un correcto diseño.

\section{Análisis dinámico en los cilindros hidráulicos de elevación}

Para el análisis del sistema de elevación y aseguramiento de la fuerza necesaria en los cilindros hidráulicos realizamos la simulación en el software ADAMS donde se puede medir la fuerza necesaria para la elevación de la máquina. En la parte de simulación se podrá medir la fuerza en el cilindro para lo cual seleccionamos el MOTION 1. Igualmente seleccionamos measure donde podemos medir la magnitud necesaria para elevar el sistema.

Figura 11. Sistema de elevación y volteo en el software ADAMS y la fuerza necesaria en el cilindro de elevación

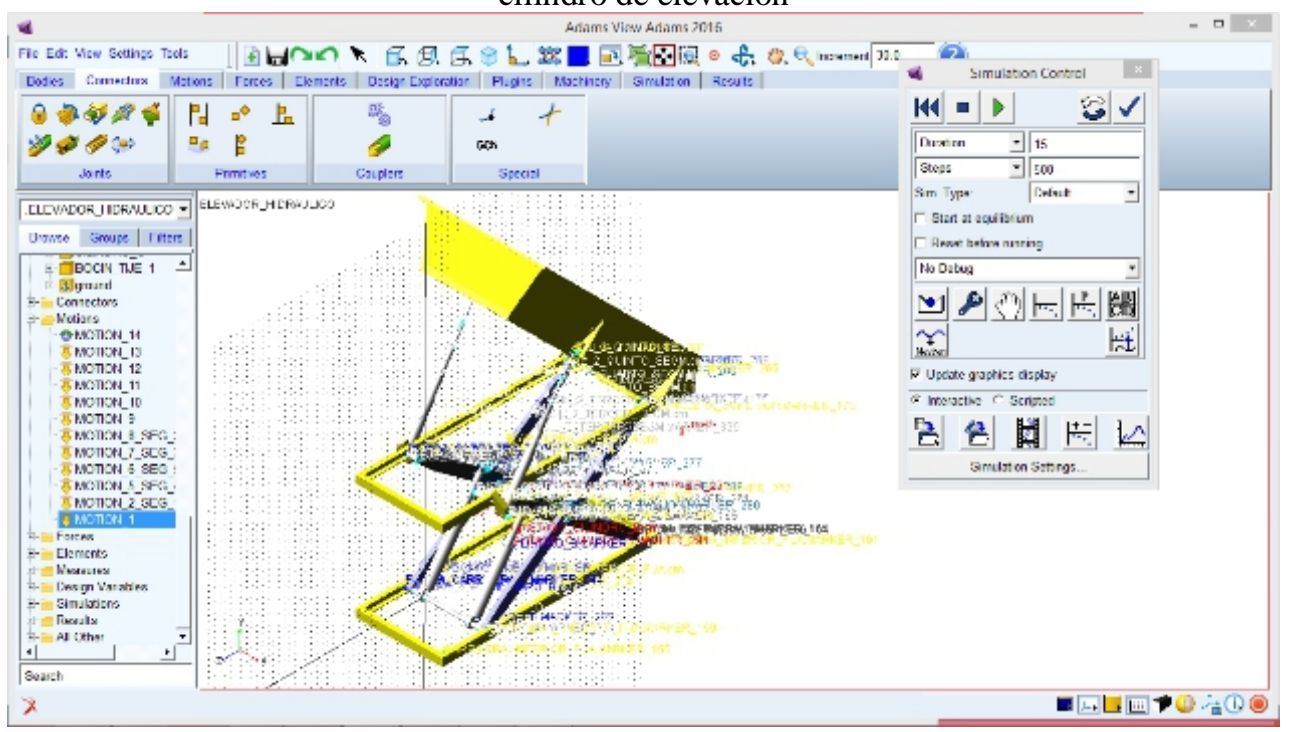

Fuente: Adams, 2018

Realizado por: Los autores. 


\section{Análisis dinámico del elevador hidráulico en las trayectorias curvas}

Una vez realizado el diseño del elevador hidráulico estático y dinámico procedemos a realizar la simulación de la máquina para comprobar el sistema de giro de las llantas en el software MSC ADAMS, donde se determinó la velocidad máxima de $36 \mathrm{~km} / \mathrm{h}$, velocidad a la que puede ser trasportado el elevador sin que se produzca un volcamiento.

Figura 12. Sistema de dirección en el software ADAMS (prueba de vuelco)

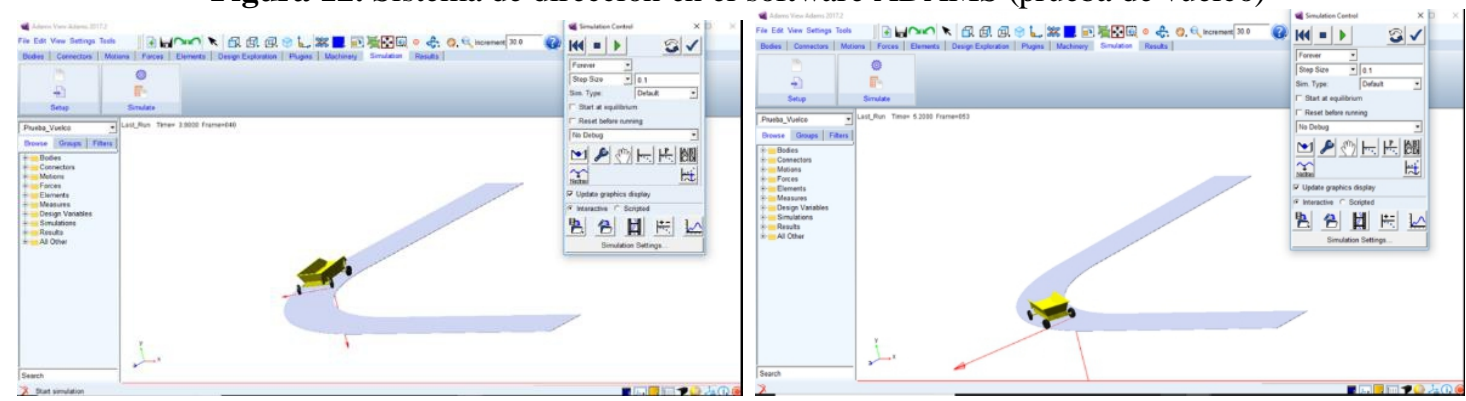

Fuente: Adams, 2018

Realizado por: Los autores.

\section{Resultados}

El plan de pruebas de funcionamiento se aplica para la verificación del buen funcionamiento del elevador hidráulico para de esta manera cumplir con los objetivos planteados, ya sea la prueba en vacío y a plena carga.

Pruebas a plena carga. Para nuestro caso se realiza la prueba en la hacienda FUSAKATAN CIA. LTDA, siendo trasladado el elevador hidráulico con un tractor agrícola hacia los sembríos de palma donde se receptará el fruto en la máquina para luego elevar y voltear al camión de trasporte que llevará el fruto hacia la planta procesadora de palma africana.

Figura 13. Prueba de funcionamiento del elevador hidráulico con volteo
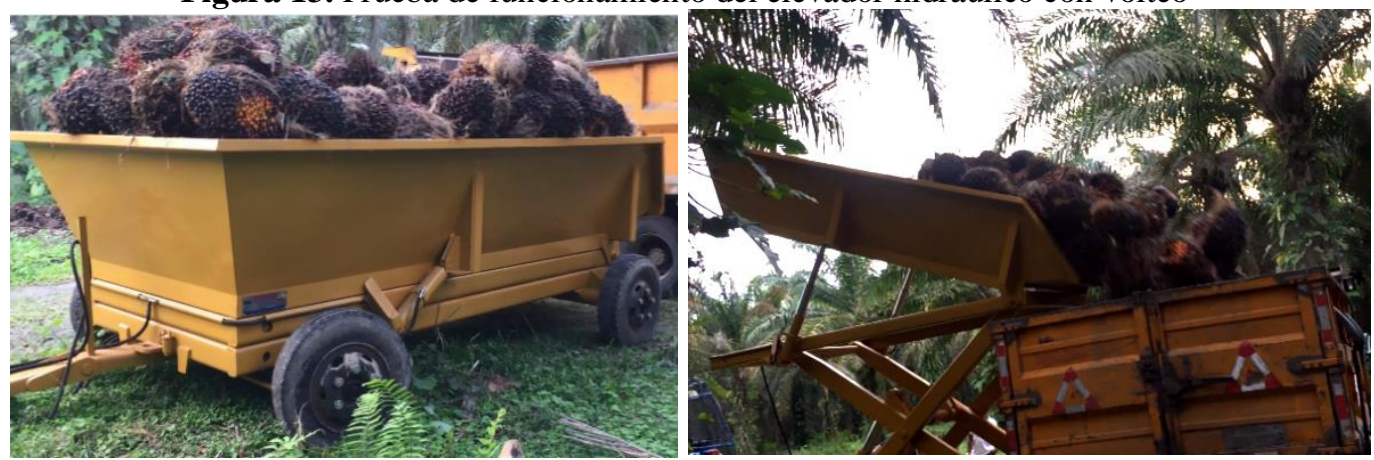

Realizado por: Los autores.

Uno de los factores es el tiempo de demora en el proceso de cosechado. Este se optimiza con el elevador llegando a 20 minutos aproximadamente en todo el proceso, tiempo después del cual la máquina esta lista para un nuevo 
cargamento. Gracias a esto se satisface la necesidad del cliente, obteniendo un fruto fresco con un mayor flujo de cosecha de la palma africana en las procesadoras industriales.

Tabla 6. Datos obtenidos a plena carga

\begin{tabular}{|c|c|}
\hline \multicolumn{2}{|c|}{ ARRANQUE A PLENA CARGA } \\
\hline Tiempo de cargado & $10 \mathrm{~min}$ \\
\hline Tiempo de parqueo. & $3 \mathrm{~min}$ \\
\hline Tiempo de elevación & $2 \mathrm{~min}$ \\
\hline Tiempo de volteo & $2 \mathrm{~min}$ \\
\hline Tiempo de bajada. & $3 \mathrm{~min}$ \\
\hline
\end{tabular}

Fuente: Investigación, 2018

Realizado por: Los autores.

Prueba de funcionamiento de transporte y dirección. Para la prueba de funcionamiento del sistema de dirección se implementó un sistema de pines y bocines que es utilizado en la industria camionera. Este sistemaes guiado por el tiro de la máquina que está anclado al tractor agrícola, estas son luego direccionadas a través de barras macizas al sistema de eje-rueda para tener facilidad de transporte del elevador.

Tabla 7. Prueba del sistema de dirección

\begin{tabular}{|c|c|}
\hline \multicolumn{2}{|c|}{ Sistema de dirección } \\
\hline Giro igual & $\mathrm{Si}$ \\
\hline Angulo & $45^{\circ}$ \\
\hline Arrastre & Mínimo \\
\hline
\end{tabular}

Fuente: Adams, 2018

Realizado por: Los autores.

\section{Discusión}

El elevador hidráulico con volteo para la cosecha de palma africana, se diseñó y se analizó de forma analítica y mediante software. El mismo fue elaborado con materiales de alta resistencia para los diferentes sistemas, dándonos un coeficiente de seguridad confiable para el proceso de cosechado. En el sistema de transporte y dirección tenemos un sistema eficientemente comprobado con las pruebas de funcionamiento en el terreno de trabajo del elevador.

\section{Conclusiones}

Se construyó el elevador hidráulico con volteo como herramienta para facilitar y mejorar la cosecha de palma africana. Como primer factor fundamental se observó que el sistema de elevación mediante los cilindros hidráulicos funcionó correctamente basándose en las pruebas de funcionamiento en vacío y a plena carga, con lo cual se puede decir que se ha cumplido con los objetivos planteados al inicio del trabajo. 
De igual manera se hicieron las pruebas de funcionamiento del elevador hidráulico con volteo y se determinó que cumple con los requerimientos planteados, mejorando el proceso de cosechado de palma africana y contribuyendo así a disminuir el tiempo de cosecha de la palma africana.

Mediante el estudio bibliográfico se determinaron igualmente las características de la palma africana, tiempos del cosechado de cada parcela, así como también la metodología empleada para la cosecha del fruto a nivel nacional y mundial.

Con las pruebas de funcionamiento realizadas se observa que el cosechado del fruto y transporte hacia la procesadora de palma africana se hace en menor tiempo, optimizando las propiedades del fruto al rato de procesarlo.

En el proceso de diseño se elaboró la matriz QFD (Casa de la calidad), donde se cumplió con la voz del usuario y los requerimientos técnicos del ingeniero. En ella se tomó en cuenta la matriz morfológica con tres soluciones para seleccionar el mejor diseño, el cual en nuestro caso es la alternativa tres pues la misma cumple con todas las especificaciones.

Para el diseño de la estructura, tolva y elementos mecánicos que conforman el elevador hidráulico se emplearon fórmulas de diseño, catálogos para la selección de componentes hidráulicos y el uso de softwares como el ANSYS donde se realizó un análisis estático del elevador y ADAMS para encontrar dinámicamente la fuerza necesaria en los cilindros hidráulicos.

Se realizó también la simulación en el software MSC ADAMS verificando que no hay ninguna interferencia de elementos en el sistema de elevación y se comprobó la fuerza necesaria en los cilindros hidráulicos tanto de elevación como de volteo para la capacidad requerida.

Se modeló todo el sistema en el software SolidWorks para garantizar que los sistemas a construir no colapsen durante las pruebas de funcionamiento y obtener los planos del elevador hidráulico para la construcción.

El prototipo del elevador hidráulico con volteo funcionó sin inconvenientes en la prueba de funcionamiento al someterle a la máxima carga del fruto de palma africana. Se pudo notar que el tractor agrícola funciona sin inconvenientes en el instante de transmitir la presión mediante el aceite hidráulico hacia los cilindros.

Se verificó en el software MSC ADAMS el sistema de dirección, el cual funcionó correctamente gracias al frontal con puntas ancladas a las barras de dirección. Estas barras brindan facilidad para el transporte del elevador hidráulico y evitan que las llantas se arrastren al momento de curvar. De igual forma se determinó una velocidad máxima de $35 \mathrm{~km} / \mathrm{h}$ para evitar el volcamiento. 
Mediante las pruebas de funcionamiento se verificó de igual manera que los sistemas de elevación y volteo funcionan correctamente, garantizando mayor rendimiento en el proceso de cosechado y transporte hacia los camiones.

\section{References:}

1. Asociación Interciencia (2008). A, Bula A, Sanjuán H. Interciencia. [Internet]. Vol. 33, Interciencia. Asociación Interciencia; [cited 2018 Apr 24]. 232-236 p. Available from: http://www.scielo.org.ve/scielo.php?pid=S0378$18442008000300015 \&$ script=sci_arttext

2. Chinchilla, CM. Manejo integrado de problemas fitosanitarios en palma aceitera Elaeis guineensis en AmŽrica Central. :69-82.

3. Eduardo Urueta Sandino (1972). Universidad Nacional de Colombia. Facultad Nacional de Agronomía EU, Sandino EU. Revista Facultad Nacional de Agronomía. [Internet]. Vol. 27, Revista Facultad Nacional de Agronomía Medellín. 1972 [cited 2018 Apr 24]. 75-77 p. Available from:

https://revistas.unal.edu.co/index.php/refame/article/view/30020

4. Fats, V. (2015). Determinantes de la competitividad del sector de Determinants of Competitiveness for the Sector: Oil Palm, Oils; 36(1):13-24.

5. Fontanilla, C., Nieto, D., \& Urueta, J. (2015). Consideraciones sobre el transporte de fruto de palma de aceite en la Zona Norte de Colombia. Rev Palmas;36(1):41-53.

6. Genty, P. (2005). Estudios entomológicos con relación a la palma africana en América Latina; 22-31.

7. Mexzón, R. (1997). Pautas de manejo de las malezas para incrementar las poblaciones de insectos beneficos en el cultivo de palma aceitera (Elaeis guineensis Jacquin). Agron Mesoam; 8(2):21-32.

8. Ocampo Valencia, S. (2018). Agroindustria y conflicto armado El caso de la palma de aceite. [cited Apr 24]; Available from: https://revistas.uniandes.edu.co/doi/pdf/10.7440/colombiaint70.2009. 07

9. PWC (2011). Ficha Sectorial ETT; Available from: https://kc3.pwc.es/local/es/kc3/pwcaudit.nsf/fichasexterna/ett?opendo cument

10. Revista de la Sociedad Entomológica Argentina (2018). [Internet]. [s.n.]; [cited 2018 Apr 24]. Available from: http://www.scielo.org.ar/scielo.php?pid=S0373$56802008000100005 \&$ script $=$ sci_arttext\&tlng=pt

11. Tecnología y logística del transporte de frutos y aceite de palma: la 
experiencia del grupo Agropalma Technology and logistics for palm oil and fruit transportation developed by the agropalma group. 2010;31:9-18.

12. Universidad del Zulia (2006). Facultad de Agronomía M, Sanabria N, Arcia A. Revista de la Facultad de Agronomía. [Internet]. Vol. 23, Revista de la Facultad de Agronomía. 2000. Universidad del Zulia; 2006 [cited 2018 Apr 24]. 420-428 p. Available from: http://www.scielo.org.ve/scielo.php?pid=S0378-

$78182006000400005 \&$ script=sci_arttext 